Canadian University Music Review

Canadian University Music Review

Revue de musique des universités canadiennes

\title{
La notion de structure chez John Cage
}

Johanne Rivest

Volume 12, numéro 1, 1992

URI : https://id.erudit.org/iderudit/1014210ar

DOI : https://doi.org/10.7202/1014210ar

Aller au sommaire du numéro

Éditeur(s)

Canadian University Music Society / Société de musique des universités

canadiennes

ISSN

0710-0353 (imprimé)

2291-2436 (numérique)

Découvrir la revue

Citer cet article

Rivest, J. (1992). La notion de structure chez John Cage. Canadian University Music Review / Revue de musique des universités canadiennes, 12(1), 37-51.

https://doi.org/10.7202/1014210ar

All Rights Reserved (C Canadian University Music Society / Société de musique des universités canadiennes, 1991
Ce document est protégé par la loi sur le droit d'auteur. L’utilisation des services d'Érudit (y compris la reproduction) est assujettie à sa politique d'utilisation que vous pouvez consulter en ligne.

https://apropos.erudit.org/fr/usagers/politique-dutilisation/ 


\section{LA NOTION DE STRUCTURE CHEZ JOHN CAGE}

\section{Johanne Rivest}

\section{Introduction}

Il est généralement admis, en ce qui concerne la musique de Cage, qu'elle est aléatoire, voire arbitraire et que la notation qu'elle emploie convie l'interprète à l'improvisation libre. Or, cette affirmation mérite quelques interrogations, car des notions complémentaires de raison-cœur, rationalité-irrationalité gouvernent à la fois la pensée de Cage et sa manière de composer. De même, on a beaucoup discuté, en regard de l'approche de John Cage, des étapes successives qui l'ont conduit à l'indétermination. Cage en a lui-même largement abreuvé ses auditeurs en écrivant divers articles et textes réunis en recueils dès 1961. Si cet abandon progressif de la structure a pu être justifié par une esthétique puisant largement aux religions orientales, le revers de la médaille, les quelques paramètres qu'une méthode compositionnelle contrôlait encore, a moins retenu l'attention. C'est cet aspect plus lié à la poiesis du compositeur que j'envisage d'approfondir.

Mon objet de réflexion étant la notion de structure chez Cage, on ne peut comprendre cette notion sans l'opposer aux trois autres qui, toutes, définissent ce qu'il entend au début de sa carrière par " composition », soit les notions de forme, de méthode et de matériau. Voici donc comment il exprime cette définition en 1949 :

Structure in music is its divisibility into successive parts from phrases to long sections. Form is content, the continuity. Method is the means of controlling the continuity from note to note. The material of music is sound and silence. Integrating these is composing. ${ }^{1}$

À cause du caractère paradoxal qu'il y a à opposer structure et forme, ce sont ces deux concepts qui retiendront mon attention, et ce, afin de cerner à quoi cela réfère dans l'esprit de John Cage, via ses propres acquis culturels, conscients ou pas. Pour aborder ces notions, je vais d'abord exposer ce qui lie et oppose

${ }^{1}$ « Forerunners of Modern Music» (1949), in Cage 1961: 62. 
Schoenberg et Cage quant à leurs définitions respectives, puis, dans un deuxième temps, comment elles sont définies dans la littérature musicologique (Hanslick, Adorno, Dahlhaus, Meyer). À la lumière de ces affirmations, je tenterai de préciser comment l'on peut définir ces concepts chez John Cage. Certaines œuvres charnières serviront d'illustration à mon propos, sans pour autant que j'en fasse des analyses systématiques.

\section{Cage et Schoenberg}

Cage a étudié avec Arnold Schoenberg en 1934, à Los Angeles. Il est donc légitime de se questionner, sinon sur l'influence directe, tout au moins sur l'impact de la pensée théorique de celui-ci sur celui-là. D'autant plus que Cage a lui-même attribué à Schoenberg son intérêt pour la structure ${ }^{2}$.

Mais Cage, dès 1949, oppose forme et structure :

Structure is properly mind-controlled. Both delight in precision, clarity, and the observance of rules. Whereas form wants only freedom to be. It belongs to the heart; and the law it observes, if indeed it submits to any, has never been and never will be written (Cage $1961: 62$ ).

Au sujet de la forme et de la structure, Schoenberg émet des opinions différentes de celles de Cage. En effet, la forme pour Schoenberg est à la fois le fruit d'une organisation et cette organisation même. Elle exige logique et cohérence, donc continuité. Schoenberg sépare cependant la définition esthétique de celle, plus technique, de la composition. Ainsi : « Au sens esthétique, le mot forme désigne une pièce organisée, c'est-à-dire dont les éléments fonctionnent comme ceux d'un organisme vivant. » (Schoenberg $1987: 15$ ).

Puis : «Les exigences fondamentales préalables à toute création d'une forme intelligible sont la logique et la cohérence. La présentation, le développement et l'enchaînement des idées doivent en effet être fondées sur des relations. "(Ibid.).

De plus, Schoenberg se préoccupe de la perception, laquelle doit être prise en charge par le compositeur. Pour lui, « ce sont les subdivisions appropriées qui facilitent la compréhension et déterminent la forme. » (Ibid.) Autre notion implicite à cette affirmation : la structure sous-tend la forme, ce que ne dément pas la pratique de Cage jusqu'à environ 1952.

2 « At the time [with modern dancers] I was interested in structure because I was fresh from working with Schoenberg. I thought that dealing with noises as I was I'd need another structure, so I found this time structure and immediately was able to give it to the dancers to work with. » Affirmation de 1965 (Kostelanetz 1988 : 191). 
Mais Schoenberg, sans l'exprimer de façon explicite, ne confond pas organisation et forme. Ainsi, la logique et la cohérence sont les prémisses d'une forme compréhensible - ce que l'on pourrait nommer éléments de la structure, en se référant à la définition de Cage - et cette forme est élaborée globalement et spontanément par le compositeur :

Bien entendu, le compositeur n'ajoute pas pièce par pièce, comme un enfant assemble des cubes de bois. Il conçoit la composition tout entière comme une vision spontanée. Puis il procède comme Michel-Ange, qui sculpta son Moïse dans le marbre sans esquisses, avec tous les détails, et forme directement son matériau. [...] Ces blocs musicaux (propositions, motifs, etc.) fourniront les matériaux pour la construction d'unités plus grandes, de différentes sortes, suivant les exigences de la structure. (Ibid. : 15-16)

Si la définition de la composition en quatre éléments de Cage doit une lointaine paternité à Schoenberg, Cage fait pourtant dériver les significations de Schoenberg vers une approche plus structuraliste. Ainsi, l'attribution de la structure à la raison et de la forme au cœur-donc, la séparation d'éléments qui, normalement, s'imbriquent, s'interpénètrent et s'influencent mutuellement - dénote un penchant pour le rationalisme du structuralisme.

Comme le souligne Arnold Whittall dans l'article qu'il consacre à la forme dans The New Grove, ce n'est que relativement récemment qu'on a envisagé la possibilité de compositions musicales opposant structure et forme ${ }^{3}$. Le structuralisme aurait servi, selon lui, à avancer l'argument selon lequel « la structure, au sens technique d'un système auto-régulateur de transformations, ne coïncide pas avec la forme " ${ }^{4}$. Il faut en conclure que «la structure doit être définie plus étroitement que la forme $»^{5}$.

Ainsi, l'organisation de Schoenberg correspondrait à la structure de Cage, c'està-dire à la technique de composition; car Cage, en tant qu'élève de Schoenberg, subit l'influence de sa notion organisationnelle de structure, où le tout renvoie aux parties et vice versa. Quant à la forme spontanée de Schoenberg, elle est associable à la forme de Cage, c'est-à-dire au domaine de l'instinct dans le cas de Schoenberg, et du cœur dans celui de Cage. Voilà pour les affinités entre Schoenberg et Cage. Mais les moyens de réaliser la structure divergent :

3 « It is only relatively recently that the possibility of musical compositions displaying structure as opposed to form has been envisaged. » (Whittall $1980: 710$ )

4 «[...] structure in the technical sense of a self-regulating system of transformations is not coincident with form (selon Piaget : Le structuralisme, 1968)». (Ibid.)

5 «A structure in this sense 'must be defined more narrowly than form' (Piaget)». (Ibid.) 
harmoniques dans l'enseignement que prône Schoenberg, ils sont rythmiques chez Cage, et ce, afin d'intégrer les bruits de l'environnement à la musique. Plus tard, Cage déplacera l'élaboration de la structure vers l'interprète, en abandonnant la mesure temporelle des événements et, surtout, la mesure proportionnelle reliant les parties au tout. Le concept de structure ne meurt donc pas pour autant.

En guise d'exemple, songeons à l'élaboration des structures rythmiques, ou temporalité mesurée, qui sert de cadre à l'indéterminé du matériau dans 4'33"de 1952, quoique cette œuvre puisse durer n'importe quel laps de temps, comme plus tard O'00" (1962), Variations III (1962-63), Variations IV (1963). Ce sont des œuvres que Cage appelle indeterminate of its performance, ce qui est différent des œuvres où le compositeur a tout élaboré grâce au hasard, mais qui ne laissent pas à l'interprète la possibilité de structurer lui-même certains paramètres, si ce n'est la liberté de ne pas jouer toutes les notes, comme dans Music of Changes (1951). Quoi qu'il en soit, la structure équivaut toujours au poiesis, au faire, que ce soit par le compositeur ou par l'interprète.

Avant d'observer comment cette séparation des éléments conduit Cage à les éliminer ou à en modifier le rôle dans ses activités de compositeur, continuons le tour d'horizon musicologique proposé, pour bien comprendre ces notions en général. La notion de forme sera d'abord examinée, tant du point de vue esthétique que gestaltiste ou technique.

\section{Forme}

La forme audible est un élément qui se situe du côté de la perception, psychologique ou esthétique. Il s'agit d'une notion abstraite, comme l'est la forme d'une sculpture, d'un dessin ... Plus qu'un contour, la forme est la totalité perceptible de ce qui est, de ce qui se présente à la perception, à l'appréhension. La forme ne peut être perçue que comme un tout, une chose close, du moment qu'on la contemple - même une forme ouverte, ou un work-in-progress.

Mais la forme en musique cause problème, puisqu'elle ne peut s'appréhender d'un seul coup. La musique se déroule en effet dans le temps, contrairement au domaine pictural qui se donne à voir dans son ensemble. Lorsque Cage abandonne la mesure des durées, du tout comme des parties, c'est qu'il assimile la musique à un art spatial autant que temporel. La vitesse de déplacement dans ces espaces que sont à la fois le graphisme de la notation et l'environnement acoustique devenant aléatoire, la mesure du temps n'est plus qu'un facteur secondaire, presque négligeable. 


\section{Définitions}

André Hodeir, dans le petit « Que sais-je ? » intitulé Les Formes de la musique, tente de définir distinctement genre, style, forme et structure (Hodeir 1960 : 711). Son entreprise est trop sommaire pour être réussie, car son étude propose bien plus une description des genres qu'un réel approfondissement de la notion de forme. Il a cependant le mérite d'opposer des termes qui prêtent souvent à confusion, si ce n'est à dissension. Du côté des dictionnaires et encyclopédies, la forme est un élément qui s'associe au genre. Tandis que, comme nous l'avons vu, Schoenberg associe la forme à la structure. Chez Hanslick et les formalistes, la forme s'assimile au contenu. En effet, dit Hanslick, « En musique, il n'y a pas de contenu opposable à la forme, parce qu'il n'y a pas de forme séparable du contenu »(Hanslick 1986: 163). Meyer, qui puise son inspiration du gestaltisme, assimile la forme au genre, qu'il oppose ensuite au style, comme nous le verrons plus loin.

Ce qui semble clair est que la forme a partie liée avec la temporalité, c'est-à-dire qu'elle se définit dans le temps en se détachant de l'écoulement normal du temps. En fait, elle est une sorte de résultat objectif, c'est-à-dire observable, d'un effort créateur. Selon Adorno,

L'idée de forme souligne la brutale antithèse de l'art et de la vie empirique [...]. La forme est la cohérence des artefacts - aussi antagoniste et brisée soit-elle-par laquelle toute œuvre d'art qui réussit se sépare du simple étant. (Adorno 1982 : 190-91)

Adorno ne dissocie pas, quant à lui, contenu et formé de la forme $e^{6}$. Il affirme plutôt "que la forme qui est donnée au contenu est elle-même un contenu sédimenté ... » (Ibid. : 194). Ou encore : «La difficulté à isoler la forme est conditionnée par l'imbrication de toute forme esthétique et du contenu; elle n'a pas à être conçue seulement contre lui, mais au travers de lui ... » (Ibid. : 189).

Quant à Carl Dahlhaus, même s'il qualifie l'expression «forme " d'ambiguë (Dahlhaus $1987: 248$ ), il l'associe au concret, à l'appréhension perceptive de ses qualités sensibles ${ }^{7}$. C'est ce qui l'amène d'ailleurs à distinguer «forme » de «structure » :

\footnotetext{
6 « Mais le formé et le contenu ne sont pas des objets extérieurs à la forme; ils sont les impulsions mimétiques attirées vers ce monde des images qu'est la forme; les équivoques nombreuses et nuisibles du concept de forme proviennent de son ubiquité qui conduit à appeler forme tout ce qui est artistique dans l'art. » (Adorno 1982 : 191)

7 « Categories of musical form refer to things that are real, to melodic-rhythmic forms, not to abstract components. » (Dahlhaus 1987 : 257)
} 
... the expression 'structure' can be related to abstract components, to pitches or durational values separated from the remaining sound qualities. [...] What is meant by the term 'form', on the other hand, is a concrete musical shape in which pitch, duration, dynamics and timbre interact. (Ibid. : 260)

De plus, Dahlhaus distingue ces deux termes en les campant dans les deux pôles opposés de la tripartition ${ }^{8}$, en assimilant la méthode à la structure : la structure serait le fait du compositeur, de sa technique, et la forme concernerait plutôt l'auditeur, l'appréhension globale.

... structure tends to be a technical concept, which suggests the genesis of a work, the process of production, whereas form is an aesthetic category which refers to the result, the audible shape. A structure need not - be perceivable; the method need not - be apparent from the result. The idea of an inaudible musical form would be a contradiction in terms. Structure is the aspect of the work directed at the composer, form that which is directed at the listener. (Ibid. : 260-61)

Ainsi, dans l'idée de « forme ouverte », où des sections pré-fixées sont agencées de façon variable par l'interprète, la variabilité est esthétiquement une fiction. Pour l'auditeur, dit Dahlhaus, elle n'existe pas'.

Quant à la méthode de Cage, Earle Brown et « compagnie », où le compositeur laisse à l'interprète le soin de structurer les détails, Dahlhaus éprouve de la difficulté à en accepter la conséquence ultime, qui serait, selon lui, que l'évolution de l'activité musicale à la production de l'œuvre, de la praxis à la poiesis, a été déjouée $^{10}$. Cette tendance favorable aux œuvres ouvertes est, selon lui, une marque de protestation contre les schémas fixes dont la théorie des formes serait responsable. Car, parmi les deux principes que la théorie formelle du XIX ${ }^{e}$ siècle

${ }^{8}$ La tripartition sémiologique, telle que définie par Jean-Jacques Nattiez dans Musicologie générale et sémiologie (1987 : 34), caractérise trois dimensions du phénomène symbolique : le poḯtique, où « la forme symbolique résulte d'un processus créateur qu'il est possible de décrire ou de reconstituer »; l'esthésique, qui correspond à " un processus actif de perception "; le niveau neutre, qui est la manifestation physique et matérielle « sous l'aspect d'une trace accessible aux sens ».

9 « For the listener it does not exist; he does not relate the version he is hearing to other possible ones the performer could have chosen, but did not choose. What is a variable form on paper is fixed in performance; and, insofar as form is a category that refers to the perceivable result and not to the method, 'open form' is not 'open'. » (Dahlhaus 1987 : 261-62)

10 « The ultimate consequence would be the assertion that the evolution from musical activity to producing works, from praxis to poiesis, has been misguided. Yet it is difficult to accept this view. » (Ibid. : 262) 
véhiculait, l'appartenance de la forme musicale au genre pose problème au $\mathrm{XX}^{\mathrm{e}}$ siècle. L'autre principe était la distinction, sur le plan esthétique, entre contenu et forme. Même si ces deux principes se trouvent abandonnés au $\mathrm{XX}^{\mathrm{e}}$ siècle, certains problèmes qui leur sont reliés persistent. Ainsi, une théorie du nouveau et de la nouvelle musique devrait développer, toujours selon Dahlhaus, une poétique musicale non divisée entre théorie de la forme et esthétique, comme

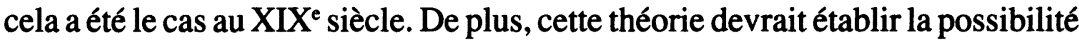
d'apprécier les formes musicales sans évoquer les concepts de forme. ${ }^{11}$

Si le concept de forme perçu comme schéma fixe est rejeté par Cage, c'est au profit du processus, où la notion de temporalité perd de sa finalité. C'est la téléologie, la directionalité qui est écartée. Cependant, comme le précise Dahlhaus (1987 : 220), le concept d'œuvre, donc de forme close, a toujous été précaire depuis son arrivée tardive dans l'histoire. " For music is directly and primarily experienced as a process or a performance, and not as a form which confronts the listener » (Ibid.). Cette notion de processus est donc déjà inhérente à la perception musicale, ce qui complique encore la définition du terme « forme ». Autre ambiguiité : le refus de la forme s'exprime par une attention agrandie au moment présent plutôt qu'à la continuité. En effet, la musique informelle nie la forme en niant la temporalité. Seul compte le moment présent. Il s'ensuit une perte de lien entre passé, présent, futur et, paradoxalement, une perpétuité de la continuité, laquelle n'a ni réel commencement ou fin, si ce n'est que des contingences matérielles bien particulières. Ainsi, le découpage temporel n'est pas effectué en vue d'une finalité, mais montré comme une tranche d'un organisme quasi vivant, auto-régulateur. ${ }^{12}$ Cage, à plusieurs reprises, définit le processus comme événement sans temporalité, où la notion d'unicité du moment est de première importance.

Pour Meyer, la compréhension de la forme sera plus aiguisée si le style est compris. ${ }^{13} \mathrm{C}$ 'est qu'il assimmile le genre à la forme; ainsi, une forme sonate du

11 « As long as one understood forms in terms of genre it was not difficult to justify the assertion that a work was formless; the criticism may have been narrow-minded, but it was not unfounded. If, however, a work is an individual without genre, then the judgment seems to be based on nothing. Nonetheless, no one doubts that one can distinguish between successful and unsuccessful forms; and the difference that exists must also be explainable. » (Ibid. : 259-60)

12 Au sujet de Roaratorio, Cage déclare en 1981 : « We put as many sounds as we could in the space of a month. And then we stopped arbitrarily because had we continued and recorded every sound, we would probably still be working. » (Kostelanetz 1988 : 167)

13 « ... if we do not understand a given style, if we lack the proper habit responses, we will either fail to apprehend shape or, if we apprehend it in terms of another style, we will fail to comprehend it. " (Meyer 1956: 160) 
style classique est un genre qui permet d'identifier le plan formel qui lui est associé, par opposition à une sonate du $\mathrm{XX}^{\mathrm{e}}$ siècle (par exemple, chez Boulez ou même Cage).

Mais si un style n'admet pas l'analyse, à cause de certains facteurs inhérents qui l'excluent, la musique ne pourra être évaluée. Ainsi,

... the evaluation of truly aleatory music is not merely problematic in practice; it is impossible in principle. For since successive sounds [...], whether notated or recorded, are by definition unrelated to one another, what is cannot be distinguished from what might have been. [...] since the specific sound successions generated by random means are not consequences of human choice, they are not [...] «stylistic ». Rather, like phenomena in the natural world, they simply exist. (Meyer $1979: 41-42$ )

Car un style est déterminé par une volonté, il cherche la communication et est donc porteur de sens. Or, l'aléatoire, en évacuant l'intentionalité, nie le sens (ou contenu). Cependant, comme Meyer établit une hiérarchie entre style, règles et stratégies, il finit par concéder aux choix aléatoires de Cage un style.

\begin{abstract}
Aleatory composers make choices: not on the level of choosing successive sound-relationships within works, but on the level of precompositional constraints: rules (that the work be aleatory) and strategies (that randomness be generated by throwing dice, etc.). As a result it seems reasonable to argue that though style plays no role in the listener's understanding and experience of such pieces, the composer's behavior - his decision to write aleatory music and his choice of strategies for generating random successions - has style, and can for this reason be evaluated. (Ibid. : 42-43)
\end{abstract}

Il est à noter que Meyer fait une distinction entre l'expérience de l'auditeur et l'organisation du compositeur. Cela rejoint la distinction entre "formé » et « forme » proposée par Adorno. Cela exprime aussi l'importance de séparer ce qui est du domaine de l'esthésique de ce qui relève du poiétique en ce qui a trait aux éléments de forme et de structure, et d'attribuer définitivement la forme à l'esthésique et la structure au poiétique. À cause de cette redistribution, il conviendrait davantage de remplacer le mot « forme » par celui de « structure » dans l'expression « forme ouverte ».

\title{
2. Cage
}

Si l'on parcourt les écrits de Cage chronologiquement en regard de ces notions dont il se sert pour définir la composition, on peut conclure, au sujet des premières étapes de sa pensée, que Cage, après avoir assimilé la forme à l'expressivité, l'associe de plus en plus au contenu. 
... when I used the word form then [in $1937^{14}$ ], that I meant what I later called structure (the divisibility of the whole into parts). Later I used form in the sense that people generally use the word content (that aspect of composition which is best able to be free, spontaneous, heartfelt, and so on). ${ }^{15}$

Puis, à la suite de Marshall McLuhan, Cage rejette le principe de contenu.

McLuhan in his work on mass media begins by saying that content is of no importance. He says 'the medium is the message'. And he says you can come to this conclusion and this awareness only if you divorce yourself from thoughts of content. This is very similar to my statement about divorcing oneself from thoughts of intention, they go very well together. ${ }^{16}$

Ce qui frappe ici est la notion implicite que l'intention équivaut au contenu. C'est donc dire que la non-intentionalité chez Cage renvoie à la perte de contenu, et non à l'absence de matériau. C'est là, sans doute, ce qui l'oppose le plus au formalisme, lequel fait se loger le contenu dans le matériau.

Chez Cage, l'absence de contenu rend possible la superposition d'œuvres. Mais en niant le contenu et en conservant le matériau, nie-t-il la forme pour autant? Et la dissociation forme/contenu est-elle réellement possible? En d'autres termes, la non-intentionalité empêche-t-elle la forme d'exister?

Cette absence de contenu explique pourquoi Cage accordera à la notion de " choix contraint ${ }^{" 17}$ une grande importance, car, s'il n'y a pas de contenu à exprimer, comment privilégier un objet musical plutôt qu'un autre?

\section{Structure}

Pour Cage, la structure, ou plutôt son élaboration, s'apparente à une discipline, telle que mise de l'avant par le bouddhisme zen.

14 "The principle of form will be our only constant connection with the past. Although the great form of the future will not be as it was in the past, at one time the fugue and another the sonata, it will be related to these as they are to each other : through the principle of organization or man's common ability to think. » « The Future of Music: Credo » (1937), in Cage 1961: 5-6.

15 Affirmation de 1961 (Kostelanetz 1988 : 68).

${ }^{16}$ Affirmation de 1965 (Ibid. : 217).

${ }^{17}$ L'utilisation du I Ching représente ce que je nomme «choix contraint ». Comme Cage le mentionne lui-même au sujet de son recours au hasard, les possibilités statistiques sont limitées : « ... le hasard de la physique contemporaine, celui des random operations, correspond à une distribution égale d'événements. Le hasard auquel j'ai recours, celui des chance operations, est différent : il suppose des distributions inégales d'éléments. C'est ce qu'apporte le Livre chinois des oracles : le I Ching, ou les cartes astronomiques dont je me suis servi pour Atlas Eclipticalis. Je n'obtiens pas cet objet physique auquel s'intéresse le statisticien. » (Charles $1976: 74$ ) 
Ainsi, il met en œuvre des structures vides, où le contenu importe peu parce qu'il n'y a pas d'intention, où tout matériau est possible. « A structure is like a bridge from nowhere to nowhere and anyone may go on it: noises or tones, corn or wheat. Does it matter which? ${ }^{* 18}$

Même si Cage opère une segmentation dans sa théorie de la composition, ces catégories ne sont pas étanches. En effet, une structure ne peut exister sans contenu expressif. "Structure without life is dead. But life without structure is unseen. Pure life expresses itself within and through structure. Each moment is absolute, alive and significant. ${ }^{" 19}$

La structure est-elle, traditionnellement, vraiment fonction de l'harmonie ou de la tonalité, comme Cage le laisse entendre? D'autre part, est-ce vrai que la série dodécaphonique n'offre aucune structure ? « The twelve-tone row does not offer a structural means; it is a method, a control, not of the parts, large and small, of a composition, but only of the minute, note-to-note procedure. ${ }^{* 20} \ll$ The twelvetone row offers bricks but no plan. ${ }^{21}$

Tout dépend de la définition que l'on donne au mot structure. Chez les structuralistes, en effet, une structure définit un petit élément, est une petite configuration qui a une incidence sur la forme. Pour Dahlhaus, par exemple, «... the term 'structure' suggests details, connections in a small space; the word 'form', on the other hand, the outline of the whole, relations over wide stretches " (Dahlhaus 1987:260). Cela est sans doute la réponse apportée par les structuralistes à la perte de la tonalité comme ciment structurel. Quant à Cage, sa réponse à ce problème sera d'abord les structures temporelles, lesquelles accueillent aussi bien le son que le silence. C'est que Cage croit encore à cette dualité son-silence qu'il s'agit de réunir par un moyen terme qui est le cadre temporel.

Par ailleurs, Cage affirme, en 1977 :

I understand the word 'structure' as the division of the whole into parts. And I would apply the usefulness of the idea of structure to a work of art that sets

18 Affirmation de 1959 (Cage $1961:$ 124). Cette notion de la structure comme cadre où s'intègrent des formes vivantes, et donc variables, Cage en puise l'inspiration en partie dans le tala hindou. Comme dans le tala, les structures rythmiques de Cage s'appuient sur un principe cumulatif, favorisent les irrégularités métriques, déterminent des périodes plutôt que des cellules rythmiques, servent de base à la composition, délimitent des unités minimales de durée (les secondes, dans le cas de Cage), admettent des fractions.

19 « Lecture on Nothing » (1959), in Cage $1961: 113$.

${ }^{20}$ Affirmation de 1949 (Cage $1961: 63$, n. 7).

${ }^{21}$ Affirmation de 1949 (Ibid. : 64, n. 8). 
out to be an object, namely, to have a beginning, middle, and end. And if one is making a work, which I do often, that is not an object, but a process, then that concern doesn't enter ... (Kostelanetz 1988 : 217)

La nécessité de la structure rejoint ici celle d'un objet délimité dans le temps, donc « formé », au sens adornien. ${ }^{22}$

Si Cage finit par rejeter la structure, c'est qu'il découvre que le silence n'existe pas, que l'opposition son-silence est anéantie par la suprématie du silence qui est, en fait, tout son nonintentionnel, et donc qu'il n'y a plus nécessité d'intégrer, grâce à la structure, les opposés. En fait, dès 1952, avec Music for Piano, Cage affirme que la structure ne fait plus partie de ses moyens de composer. Il faut comprendre que, par " structure », il sous-entend alors la mesure temporelle en temps réel, ce à quoi il renonce pour se défaire de cette manie de tout mesurer. Mais c'est avant tout par 4'33" que Cage expose cette découverte de la nonexistence du silence. Pourtant, dans cette œuvre de 1952, l'élaboration s'est faite sur le même principe que dans Music of Changes, c'est-à-dire par des diagrammes où figuraient tous les paramètres du son, moins ceux de la hauteur, du timbre et de l'amplitude - donc, où ne subsistait que la durée. ${ }^{23}$ Une opposition persiste cependant pour le compositeur, soit celle qui se joue entre la volonté et le hasard. La structure, qui était classée du côté de la raison par Cage en 1949, fait place à la raison elle-même, ou mind, laquelle peut à son tour conditionner des éléments de temporalité (début et fin d'une pièce), la densité (comme dans Music of Changes), différents paramètres du son (ou matériau) ou encore la façon de passer d'un événement à l'autre (méthode). Et cette raison ou mind, qui détermine ce qui sera à déterminer, se sert généralement du $I$ Ching pour répondre aléatoirement à ses questions. ${ }^{24}$

${ }^{22}$ Le début de cette déclaration de Cage s'apparente assez à la définition du terme « structure » telle qu'on la retrouve dès ses débuts, au XVII ${ }^{\mathrm{e}}$ siècle, en Occident : « ... le mot désigne à la fois a) un ensemble, b) les parties de cet ensemble, c) les rapports de ces parties entre elles ... » (Bernot 1955, cité par Bastide $1972: 10$ ).

23 "When I wrote 4' 33" I was in the process of writing the Music of Changes. That was done in an elaborate way. There are many tables for pitches, for durations, for amplitudes. All the work was done with chance opearations. In the case of 4' $33^{\prime \prime}$ I actually used the same method of working and I built up the silence of each movement and the three movements add up to 4'33". I built up each movement by means of short silences put together... » (Conférences de 1988-89, réponses aux questions des étudiants, in Cage 1990 : 20-21.)

24 « Instead of representing my control, [my likes and dislikes] represent questions that I've asked and the answers that have been given by means of chance operations. I've merely changed my responsibility from making choices to asking questions. " Affirmation de 1982 (Kostelanetz $1988: 214)$. 


\section{Conclusion}

Ultimement, la désintégration de la structure - comme tout et comme parties reliées entre elles et au tout - conduit à l'abolition de la notion d'œuvre, telle que véhiculée par la culture occidentale. Pour Cage, si l'art existe, ce n'est qu'en tant que prise de conscience plus aiguë de la vie elle-même ${ }^{25}$, laquelle comporte toutes les possibilités à la fois, tel un chaos que chaque percepteur est libre d'ordonner à sa guise. La vie est donc déjà pour lui un processus, et c'est sur ce modèle qu'il calque ses œuvres. Sans réel début ni fin, surtout sans finalité, avec une attitude ouverte à toute éventualité. Mais il reste que ce simulacre de chaos, dont se composent plusieurs de ses œuvres, est sous-tendu par une répartition dans le temps et l'espace de contraintes plus ou moins strictes, régissant un nombre plus ou moins important d'éléments, mais de contraintes tout de même, qu'il met en place par les questions qu'il adresse au I Ching. La règle d'or est de ne pas respecter ce qui constitue le contenu, c'est-à-dire les relations causales, les fonctions. Perte, donc, de l'aspect sémantique de la structure. Ne reste qu'une syntaxe ordonnée arbitrairement et ce, pour permettre au cœur, à l'opposé de la raison, d'appréhender une forme, un sens, qui n'est pas du domaine de la rationalité.

Car la forme est partout, de dire Cage.

When I say that, « I am not interested in form », or « how can I use the word form ", I have to ask another question, namely, where do we see any formlessness? Particularly nowadays with telescopes, with microscopes, etc., as one of my painter friends, Jasper Johns, says, « the world is very busy $\gg$. Form everywhere. ${ }^{26}$

Ce que Cage propose, c'est l'indépendance de ces quatre éléments que sont forme - matériau - méthode - structure, même s'il leur reconnaît certains points de jonction. Ainsi, la forme n'est pas chez lui la substance, puisque le matériau, donc la substance matérielle, en demeure distinct.

Sans doute devrait-on rechercher la définition du concept de forme chez John Cage dans la petite dimension (sons isolés). Différents niveaux de la forme seraient ainsi possibles : car, de plus en plus, chez Cage, la forme se réduit au son

25 «... art is not an escape from life, but rather an introduction to it. » Affirmation de 1966 (Ibid. :

211).

${ }^{26}$ Affirmation de 1961. « Interview with Roger Reynolds » (Dunn $1962: 50$ ). 
lui-même, à sa morphologie. ${ }^{27}$ En guise de prémisse, il y a l'invention du piano préparé, en 1938, qui isole chaque son en lui attribuant un timbre spécifique.

Quant à la structure, elle n'est pas issue du matériau, ni de la méthode : dans une œuvre sérielle, les sons ont une fonction structurelle importante, puisque les relations qu'ils forment coordonnent la structure même; tandis que les sons de la musique expérimentale font partie du domaine empirique. Ils ne sont pas émis par une volonté structurante, ni dans un but avoué de cohérence.

En définitive, la proposition énoncée par Cage en 1949 demeure pertinente. Pourtant, puisque la forme ne saurait se définir sans avoir recours au matériau, et que la structure ne peut être engendrée indépendamment de la méthode (car comment apercevoir une forme invisible, et élaborer une structure sans méthode ?), comment donc est-il possible d'isoler la méthode de la structure, le matériau de la forme, ainsi que l'a fait Cage?

Je crois que c'est justement cette atomisation des procédures de composition qui rapproche Cage d'un certain structuralisme. Et ce qui l'en éloigne, le processus, amène un concept qu'il faudrait étudier en rapport avec la structure, celui de la mobilité, dont Cage se sert par l'utilisation du Livre des transformations ou I Ching, en anglais Book of Changes. "La nature ne nous montre jamais que le changement [...]. Il est impossible de saisir les structures si l'on ne comprend pas les processus » (Lévi-Strauss $1972: 43)^{28}$. Car ce qui est absent de la pensée et de l'approche de Cage n'est peut-être pas tant la structure elle-même que son immuabilité, c'est-à-dire qu'elle doive toujours figurer comme élément premier de la composition. Le « toujours » est remis en cause par Cage, car ce qui compte avant tout pour lui est la non-intention. Et la non-intention permet à la structure d'être ou de ne point être. Cela dépend presque de la distance entretenue avec le phénomène. Se référant à la nature, Cage dit :

Je me suis aperçu, simplement en me promenant dans les forêts à la recherche des champignons, qu'on peut bien dire qu'il y a une structure chez

\footnotetext{
${ }^{27}$ La dernière auvre composée à l'intention de Merce Cunningham, et intitulée Sculptures musicales, ne comporte qu'un seul son à la fois, soutenu, précédé et suivi de silences. Cage dit s'intéresser maintenant à l'harmonie contenue dans le son lui-même, car « les changements, peutêtre les transitions, existent pour chaque son ». Cage, « Entrevue par Georges Nicholson de John Cage », Musiciens par eux-mêmes, Société Radio-Canada, 16 septembre 1990.

${ }^{28}$ Lévi-Strauss cite Vogt 1960 : « Or, nous avons commis la faute de concevoir les structures comme statiques, faisant ainsi du changement, soit un principe hétérogène, soit un phénomène interne, mais de nature pathologique. Il faut, au contraire, poser le primat du changement, et considérer la structure comme la manière dont se traduit, pour l'observateur, une appréhension instantanée et artificielle d'une réalité mouvante. »
} 
certaines espèces de champignons. Une certaine structure... ou un certain art! Et l'on peut à partir de là considérer la structure et l'organisation comme très importantes. Mais si l'on observe tout, toute la journée, toutes les expériences, alors on ne peut plus parler de l'organisation! Alors l'art ou la structure s'estompent ... ${ }^{29}$

\section{RÉFÉRENCES}

ADORNO, THEODOR W.

1982: Théorie esthétique. Paris : Klincksieck.

BASTIDE, ROGER, édit.

1972: Sens et usages du terme structure dans les sciences humaines et sociales. Paris : Mouton.

BERNOT, M.L.

1955: «Contribution à l'étude internationale des Structures Sociales », Bulletin International des Sciences Sociales, VII, 4.

\section{CAGE, JOHN}

1961 : Silence. Middletown (Conn.) : Wesleyan University Press.

1990 : I-VI. Cambridge (Mass.) : Harvard University Press.

CHARLES, DANIEL

1976: Pour les oiseaux. Paris : Belfond.

DAHLHAUS, CARL

1966 : « Form », Darmstäder Beiträge zur Neuen Musik, 10, Mainz. (Repris dans Dahlhaus 1987 : 248-64.)

1987 : Schoenberg and the New Music, Essays by Carl Dahlhaus (D. Puffet et A. Clayton, trad.). Cambridge University Press.

DUNN, ROBERT, édit.

1962 : John Cage (catalogue des œuvres). New York : Peters, Henmar Press.

HANSLICK, ÉDOUARD

1986: Du beau dans la musique. Paris : Christian Bourgois, Musique/Passé/ Présent.

HODEIR, ANDRÉ

1960 : Les Formes de la musique. Paris : P. U. F., «Que sais-je? », nº 478.

KOSTELANETZ, RICHARD

1988 : Conversing with John Cage. New York : Limelight.

${ }^{29}$ Entretiens avec Daniel Charles, réalisés entre 1968 et 1972. (Charles $1976: 37$ ) 


\section{LÉVI-STRAUSS, CLAUDE}

1972: «Les limites de la notion de structure en ethnologie », Sens et usages du terme structure dans les sciences humaines et sociales (R. Bastide, édit.). Paris : Mouton.

\section{MEYER, LEONARD B.}

1956 : Emotion and Meaning in Music. Chicago : University of Chicago Press.

1979 : « Toward a Theory of Style », The Concept of Style (Lang, Berel, édit.). University of Pennsylvania Press, 3-44.

\section{NATTIEZ, JEAN-JACQUES}

1987 : Musicologie générale et sémiologie. Paris : Christian Bourgois, Musique/ Passé/Présent.

\section{SCHOENBERG, ARNOLD}

1987 : Fondements de la composition musicale. Paris : J.C. Lattès.

\section{VOGT, EVON Z.}

1960 : "On the Concept of Structure and Process in Cultural Anthropology ", American Anthropologist, 62, 1.

\section{WHITTALL, ARNOLD}

1980 : «Form », The New Grove Dictionary of Music and Musicians (S. Sadie édit.), vol. 6. Londres : Macmillan. 